\title{
Questionnaire Report on Matter Relating to Software Architecture Evaluation
}

\author{
Hassan Almari \\ Australian National University, \\ CSIT Building 108 North road \\ Canberra, ACT 0200, Australia \\ email: hassan.almari@anu.edu.au
}

\author{
Clive Boughton \\ Australian National University, \\ CSIT Building 108, North road \\ Canberra, ACT 0200 Australia \\ Chairman and Director of Software Improvements \\ GPO Box 1928, Canberra, ACT 2601, Australia \\ email: clive.boughton@anu.edu.au
}

\begin{abstract}
Evidence of the relationship between software architecture including it's styles/patterns and quality attributes continues to grow, but largely remains an art rather than a science in terms of being able to predict a relevant architecture from (known) quality attributes.

The aim of this paper is to point out those aspects that influence utilization of software architecture artefacts and their evaluation by software developers, and is part of a continuing study the results of which are intended to aid professional software/system developers with their decisions surrounding choice of (concrete) software architectures.

The earlier part of the study produced an analysis report based on a survey titled "Questionnaire on matters relating to Software Patterns - 2012". The survey was issued to software developers possessing more than 5 years experience, and produced significant results which, in turn, led to the need for this second survey. Ninety seven $(97 \%)$ percent of the participants, from six different nations, answering the first questionnaire supported this further investigation.
\end{abstract}

Index Terms-Software Architecture, Quality Attributes, Evaluation, Utilization.

\section{INTRODUCTION}

$\mathrm{T}$ HE utilization of software/system architecture and associated evaluation methods in industry is influenced by a number of factors. Varied studies have tried to detect, examine, assess, and describe the link between software architecture (SA), and quality characteristics/attributes, such as work done by [1], [2], [3], and [4]. Every study takes a unique focus scoped to a particular quality attribute or a view of the architecture. In order to discover more about these factors that influence the relationship between SA and QAs, a questionnaire was produced to target experienced software/system developers to determine their views on the applicability of existing SA artefacts and their associated evaluation methods, as well as to identify the challenges to utilization of these artefacts and methods in the development lifecycle. This paper summarises the result from the second quastionnare on matters relating to Software Architecture Evaluation (SAE). The analysis of the responses to the survey was performed using the Statistical Package for the Social Sciences (SPSS) application. These facts have been mentioned in order to help simplify table and figure symbolizations. In this paper, the use of figures and tables have been minimized due to page limitation. Full analyses of the survey (311 pages) is available upon request, through contacting the authors via email.

\section{ANALYSIS PROCEDURE}

The questionnaire surrounding SAE comprised of 23 questions which were grouped into various sections such that each section focussed on a specific objective as described below.

The first section of the questionnaire consisted of five questions aimed at gaining better background knowledge of the participants. In the second section, a further five questions focussed on the factors that affect utilization of SA modeling techniques. This was incorporated in an effort to make the utilization of SA artefacts together with all its modelling descriptions a cost-effective and time-saving process. The final section focused mainly on SAE and its challenging factors.

In analyzing the responses both one dimensional analysis and two dimensional analysis were used. In one dimensional analysis, the responses to each of the 23 questions were analyzed individually and the results were illustrated using proper statistical methods, depending on the nature of each question. In order to draw more vivid conclusions, the dependencies between different SA utilization factors were also analysed. This was achieved by carrying out a two-dimensional analysis, where some of the questions were analyzed as pair combinations in order to satisfy some of intended objectives by researchers. The methods used for both one and two dimensional analysis are described in the next section.

\section{RATIONALE OF THE ANALYSIS METHOD SELECTED}

Several methods were used to analyse survey responses. The rationale for the methods used is given below:

\section{A. Individual (One dimensional) analysis methods}

For individual or one dimensional analysis, the method of analysis used depended on the type of questions being asked. Questions which required respondents to select a single response from a list of choices, such as Q1, were analysed by presenting all responses on a single bar chart showing the total count for each choice. The bar chart was derived 
from a frequency table which was used to represent the same information as percentages.

'Descriptive statistics' was the method used to analyze questions for which responses were divided into categories without numerical values. The results were presented using a frequency table to show the percentage for each respondent as well as the cumulative percentage of the responses. In addition to the frequency table, for questions which required the respondent to make a choice from numerical responses, a Chi Square Test was performed to show the distribution of the responses in each category in order to draw a more accurate conclusion.

Questions which involved dichotomous variables were presented on a pie chart for easy visualization. It was convenient to do this since for these types of questions, there wasnt any overlap of responses. Thus the total percentage from all categories of responses was $100 \%$.

Responses to types of questions involving statements for which some level of the agreement was required were presented on a Likert scale to show the strength of agreement or disagreement. For these questions, the analysis was done by employing descriptive statistics. A one sample t-test was performed on the resultant mean for each of the questions in order to determine and confirm the significance of the concluding agreement level.

\section{B. Multi-dimensional analysis methods}

Multi-dimensional methods involving analysis responses to questions as a block, or in matrices, were employed to determine whether a relationship existed between the responses of the various combinations of questions chosen. The analysis methods included sample t-tests, cross tabulation, Chi square tests, Tukey's? HSD method, scatter plots, descriptive statistics and bar charts among others. Cross tabulation was preferred in most cases since most of the questions involved a cardinal scale, with only four questions lying on the ordinal scale. Ttests were used to analyze the latter category.

Three-dimensional and four-dimensional analyses were invalid, inappropriateness, and insignificance. The Chi-square test results for cross tabulations under three dimensional analysis are all invalid due to low cell (data) expected frequencies. Also, most of the t-tests and F-test results are all valid in statistical sense, though there were no significant results. So, they were excluded from this paper.

\section{STUDY FINDINGS}

The findings of this study uncover many factors affecting the utilization of system/software architecture artefacts by system/software developers across various sectors (work place), during the development lifecycle. The findings are summarized in this section according to the analysis method used.

\section{A. One dimensional Analyses}

This research was conducted based on responses to a questionnaire distributed to participants from various fields of expertise, the majority of whom were designers, programmers, analysts, and architects among other fields as illustrated in Table I. A majority of these professionals have gained their expertise in academia, followed by industry, and Government as shown in Figure 1.

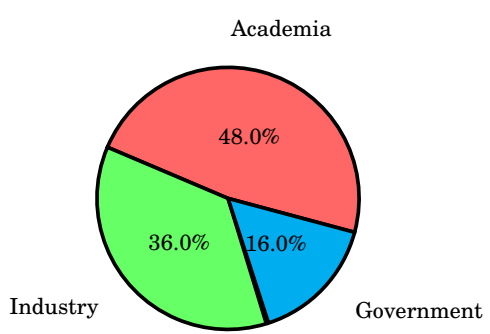

Fig. 1. Work sectors for the survey participants

The Chi-Square test confirms the significance of the results at $5 \%$ level, Chi-square $=7.84, \mathrm{p}$-value $<.05$. The findings of the research can thus be considered credible due to the varied areas of expertise of the respondents to the questionnaire.

In order to increase the reliability of the results, it was also necessary to ensure that the participants had enough familiarity or experience in a (particular) field of software/system development. The experience of the majority of the participants ranged between 5 and 10 years (48 percent), with about 30 percent having an experience of more than 15 years and $22 \%$ having experience of 10 to 15 years. Thus, the years of experience considered sufficient to gain familiarity with the trends in software/system development.

More than 83 percent of all the participants indicated that they were aware of software/system architectural description/ modelling languages, which increases the reliability of the responses given in the questionnaire, because they do have knowledge about the survey field, which help them to select a proper answers based on their experiences. At the same time, considering the experience levels, the finding raises a question about 17 percent of the participants in the software/system development industry who were not aware of software/system architectural description/ modelling languages, which could be affected by different factors such as the lack of teaching SA in academic institutions.

Despite the majority awareness of software/system architectural description/ modelling languages, an alarming 50 percent of the respondents either used models infrequently or did not use models at all. This justified the need for a further questionnaire to unearth the reasons that could be encouraging or discouraging the use of system/software architecture modelling languages.

The two major (out of 8) factors that encourage the utilization of models to describe software/system architecture as identified by the respondents include, "the ease to demonstrate the software/system concept and features" (65.2 percent), as well as its contribution in "making the designer/programmers' job easier" (30.4 percent). On the other hand, one of the two main factors (out of 5) that discourage the utilization of modelling techniques to describe software/system architecture is 
TABLE I

PERCENTAGE FREQUENCY TABLE REGARDING RESPONDENTS FIELD OF EXPERTISE.

\begin{tabular}{l||c|c|c}
\hline $\begin{array}{l}\text { What is your general field of expertise re- } \\
\text { garding software development? }\end{array}$ & Responses & $\begin{array}{c}\text { Percentage based on total } \\
\text { responses (N'=50) }\end{array}$ & $\begin{array}{c}\text { Percentage based on who } \\
\text { responds to the question } \\
\text { (N=50) }\end{array}$ \\
\hline Requirements elicitation/modelling/analysis & 23 & $46.0 \%$ & $46.0 \%$ \\
\hline Project management & 11 & $22.0 \%$ & $22.0 \%$ \\
\hline Architecture & 20 & $40.0 \%$ & $40.0 \%$ \\
\hline Design & 31 & $62.0 \%$ & $62.0 \%$ \\
\hline Coding & 29 & $58.0 \%$ & $58.0 \%$ \\
\hline Testing & 18 & $36.0 \%$ & $36.0 \%$ \\
\hline Documentation & 8 & $16.0 \%$ & $16.0 \%$ \\
\hline Other & 6 & $12.0 \%$ & $12.0 \%$ \\
\hline
\end{tabular}

"the difficulty in integrating these models with other artefacts (e.g Design models)", which makes them standalone models rendering them less useful in the process of software/ system development". The other factor is "the lack of standardization between the existing architecture modelling techniques, notations and semantics", among other factors.

In an effort to determine the best language to use to describe software/system architecture in order to increase its usefulness to all stakeholders as well as the ease of qualitative and quantitative assessment, it was found out that a combination of semiformal language and natural language used together would be preferred by the majority ( 52.2 valid percentage). Semi-formal language alone is the second most preferred (17.4 percent valid), while 13 percent prefer all three languages (formal, semi-formal and natural languages). The results above were expected, based on the researcher's observations to several current projects documentations, within the Royal Saudi Air Force (RSAF). We think its a good combination to have semiformal and natural language, due to the ease with which nondeveloper stakeholders can understand these languages as well as the ease of the evaluation processes.

There is a general agreement that developing software/system architecture using current architectural frameworks (e.g. ISO/IEC 42010, DODAF, RUP/4+1) increases the reliability, standardisation, and reusability of the resulting architecture. On the other hand, there is neither agreement nor disagreement about whether "the usage of software style/pattern concepts \& models during architecture development increases the utilisation of modelling description languages, BUT decreases the simplicity of the architecture evaluation or not". This is confirmed by a one-sample t-test as described in Table II.

In an attempt to gather the level of awareness surrounding system/software architectural metrics, an overwhelming 85 percent denied having any awareness about any system/software architectural tactics or metrics that have been or are being used for evaluating architecture description models, (e.g. detecting attacks for security). This uncovers the great need to increase awareness about the existing tactics such as the effort done by [5], as well as the metrics such as the work done by [6] for security measurements, and [7] for applying metrics to assess software artefacts, and document them. Architectural evaluation methods suffer the same fate, with more than 89 percent of the respondents lacking awareness about any architectural evaluation method that can produce quantitative measures surrounding architecture characteristics. The questionnaire therefore attempted to uncover the reasons that may be encouraging or discouraging this knowledge and/or use of software/system architectural evaluation methods.

According to the statistics gathered from the questionnaire, the two most important factors that could support quantitative evaluation for any software architecture (SA) include "availability of tools for describing and evaluating SA" (identified by 44 percent of the respondents) and "the use of standard language and architecture framework for describing SA" (identified by 40 percent of the respondents). On the other hand, two of the most important factors that hinder quantitative evaluation for any SA include the "formality level of SA description" as identified by more than half of the respondents, followed by "the language used for describing the SA" as identified by $40.5 \%$ of the respondents. These are the factors that need to concentrate on in order to improve the knowledge and use of system/software architectural artefacts and its evaluation methods that can produce qualitative and quantitative results.

The general agreement to the suggestions given by researchers in this questionnaire regarding important matters affecting architectural evaluation, and how we could aid resolving these issues, was measured as shown in Figure 2.

According to the analysis, there was a significant agreement to all the statements in Figure 2, except on the statement

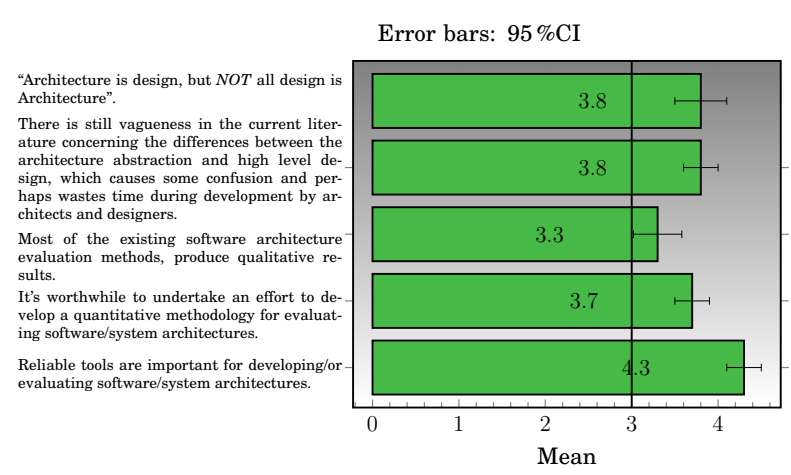

Fig. 2. Means for the five statements included - with error bar and assumed mean line. 
TABLE II

ONE SAMPLE T-TEST RESULTS FOR SPECIFIED MEAN VALUE REGARDING THE TWO STATEMENTS INCLUDED

\begin{tabular}{|c|c|c|c|c|c|c|}
\hline \multirow{3}{*}{ Items } & \multicolumn{6}{|c|}{ Test Value $=3$} \\
\hline & \multirow[b]{2}{*}{$\mathrm{t}$} & \multirow[b]{2}{*}{$\mathrm{df}$} & \multirow[b]{2}{*}{$\begin{array}{c}\text { Sig. } \\
\text { (2-tailed) }\end{array}$} & \multirow[b]{2}{*}{$\begin{array}{c}\text { Mean } \\
\text { Difference }\end{array}$} & \multicolumn{2}{|c|}{$\begin{array}{l}95 \% \text { Confidence Interva } \\
\text { of the Difference }\end{array}$} \\
\hline & & & & & Lower & Upper \\
\hline $\begin{array}{l}\text { Developing software/system architecture using current archi- } \\
\text { tectural frameworks (e.g. ISO/IEC } 42010 \text {, DODAF, RUP/4+1) } \\
\text { increases the reliability, standardisation, and reusability of the } \\
\text { resulting architecture. }\end{array}$ & 4.14 & 44 & .000 & .47 & .24 & .69 \\
\hline $\begin{array}{l}\text { Usage of software style/pattern concepts \& models during ar- } \\
\text { chitecture development, increases the utilisation of modeling } \\
\text { description languages, BUT decreases the simplicity of the } \\
\text { architecture valuation. }\end{array}$ & .00 & 45 & 1.000 & .00 & -.25 & .25 \\
\hline
\end{tabular}

that "most of the existing software architecture evaluation methods, produce qualitative results", to which the majority of the respondents remained neutral. However, the one-sample t-test on the agreement to that statement $(\mu>3)$ is statistically significant at $1 \%$ level, $\mathrm{t}=2.46, \mathrm{p}$-value $<.01$ implying a general agreement to the statement.

Finally, the agreement to the statements in Figure 3 were also tested using descriptive statistics.

From the figure it is clear that there was a general agreement to the first three statements presented. However, in regard to the last statement, about one third of the respondents (14 out of 43) either disagreed or strongly disagreed to the statement that stated that "current technology allows us to develop general software evaluation models that assess any software architecture against any quality attributes". According to a continuing study done by the researchers since 2010, we believe that there is no existence of such a tool yet. However, the current technologies are becoming closer to achieve the above statement, and many gaps have been closed during the last decade by improving SA description languages and tools. Furthermore, the above results indicated that more research needs to be done to improve and contribute to SA evaluation filed.

In addition, a significant number of the respondents (18 out of 43) representing 42 percent remained neutral regarding the statement and only $26 \%$ (11 out of 43 ) either agreed or strongly agreed to this statement. The responses were mostly

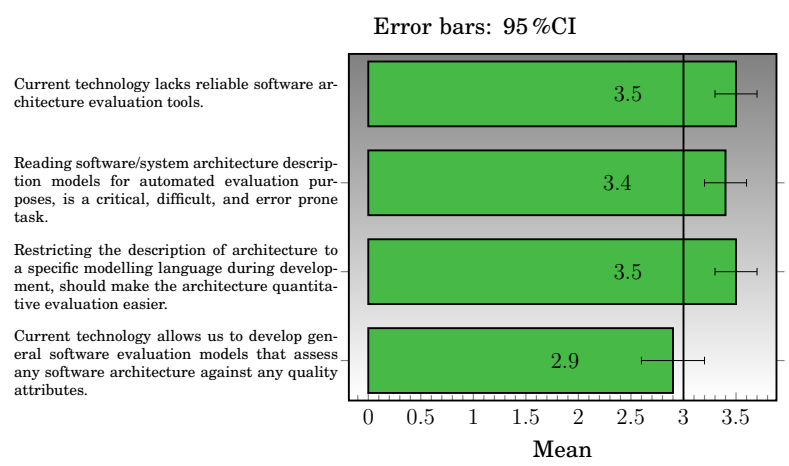

Fig. 3. Means for the four statements included - with error bar and assumed mean line. skewed around the neutral axis and a one-sample t-test on the agreement to the statement $(\mu>3)$ showed significance at $5 \%$ level with $\mathrm{t}=-.80, \mathrm{p}$-value $>.05$, indicating a general disagreement to the statement.

\section{B. Two dimension Analyses}

In order to establish the credibility of the responses as well as important trends in the responses given to the questionnaire, the questions were cross-tabulated in pairs to determine the association between each question in the pairs. This section summarizes the findings.

It was noted that the general field of expertise regarding software development did not have any association with most of the other questions in the questionnaire. However, it was noticed that the most of the respondents whose field of expertise was Project management did not think that most of the existing software architecture evaluation methods, produce qualitative results, compared to other areas of expertise $(\mathrm{t}=-$ 2.35, p-value $<.05$ ). On the other hand, Architects, more than other fields of expertise, tend to think that "Architecture is design, but NOT all design is Architecture" ( $\mathrm{t}=2.20$, $\mathrm{p}$ value $<.05)$. Furthermore, there is significant inequality in means between Architects and experts in other fields regarding the statement that "Architecture is design, but NOT all design is Architecture" ( $\mathrm{t}=2.20$, p-value $<.05)$. This shows that Architects embrace this statement more than developers in other areas of expertise. Whereas, those whose field of expertise is coding tend to disagree more than others to the same statement above, which is very important indication to the variation in understanding the architecture concept between both architects and programmers. As a result, architectural artefacts utilisation by programmers could be decrease due to their understanding of SA concept, which we believe its not correct.

Regarding software evaluation methods, experts in Project Management showed a much less significant result compared to those who have other expertise on the statement that "Most of the existing software architecture evaluation methods produce qualitative results". While, designers showed a significantly less population mean than other experts to the same statement, $(\mathrm{t}=-2.10, \mathrm{p}$-value $<.05)$. However, they also showed a greater population mean on the statement that "Reliable tools are important for developing/or evaluating software/system architectures" $(\mathrm{t}=2.04, \mathrm{p}$-value $<.05)$. 
On the other hand, experts in Coding showed a less significant population mean on the statement that "It's worthwhile to undertake an effort to develop a quantitative methodology for evaluating software/system architectures" ( $\mathrm{t}=-1.87$, $\mathrm{p}$ value $=.035<.05)$. These results are a clear evidence of how the field of expertise of the respondents to the questionnaire affected their opinion regarding evaluation methods used in system/software architecture.

There are many reasons that could cause the above variations, such as developers SA background knowledge, the level of developers involvement in SA evaluation process etc.

A similar variation in opinion was also noticed in matters relating to current technology as related to system/software evaluation methods. For example, the population mean for responses from experts in "Coding" to the item "Current technology allows us to develop general software evaluation models that assess any software architecture against any quality attributes" is significantly less than that of other experts $(\mathrm{t}=-2.50, \mathrm{p}$-value $=.008<.01)$. This is also the case for the population mean of responses from experts in "Testing" towards the same statement $(\mathrm{t}=-1.79, \mathrm{p}$-value $=.041<.05)$. These relationships led us to appreciate the varied nature of needs in the software/system development industry according to the expertise, hence concluding that a careful consideration about the area of expertise while implementing any suggestions offered in this study is vital to make the implementation more impactful.

The number of years of experience of the respondents was seen to affect their opinion on the statement that "most of the existing software architecture evaluation methods, produce qualitative results". Turkey's HSD method was used to determine the variation in the responses and the association that exists between the two. The analysis uncovers a significant difference (Mean difference $=1.33$, p-value $=.011_{i} .05$ ) between respondents with 5-10 years' experience and those with over 25 years' experience, with the agreement favouring the earlier group.

There is a higher rate of agreement among those who had awareness of modelling languages compared to those who didn't, on the statements that "Reading software/system architecture description models for automated evaluation purposes, is a critical, difficult, and error prone task", $(\mathrm{t}=1.9$, $\mathrm{p}$-value $=.04<.05$ ) and "Current technology allows us to develop general software evaluation models that assess any software architecture against any quality attributes", $(\mathrm{t}=2.1$, $\mathrm{p}$-value $=.03<.05$ ). We agree with the respondents for their for the first statement, but not to the second one, due the same reasons discussed earlier a round Figure 3.

There seems to be a variety of relationships between the opinions of respondents regarding qualitative and quantitative system/software evaluation methods and how often these respondents use software/system models in the description of software/system architecture. For example, there is a significant statistical difference between group means corresponding to various categories of how often respondents use models to describe software/system architecture for the item "There is still vagueness in the current literature concerning the differences between the architecture abstraction and high level design, which causes some confusion and perhaps wastes time during development by architects and designers", $(\mathrm{F}=2.7$, pvalue $<.05$ ). The group means for this item exhibit a difference among respondents who "Nearly always (>90\%)" used models to describe software/system architecture during their work compared to those who "Never" used them at all, Mean Difference $=1.2$, p-value $<.01$. Similarly, there is also a difference in the group mean for the item among those who "Nearly always ( $>90 \%)$ " used models to describe software/system architecture during their work, compared to those who "Infrequently $(<10 \%)$ " use models, (Mean Difference $=0.7$, p-value $<.05)$. The last difference is exhibited between those who "Nearly always (>90\%)" used models to describe software/system architecture during their work and those who "Reasonably frequent $(>15 \%$ and $<50 \%)$ " used, Mean Difference $=0.7$, p-value $<.05$.

Also, the test is significant for the item "It's worthwhile to undertake an effort to develop a quantitative methodology for evaluating software/system architectures", $\mathrm{F}=2.7$, p-value $<4.05$. So, the group means corresponding to the categories of how often respondents used models to describe software/system architecture during their work are statistically different for the item "It's worthwhile to undertake an effort to develop a quantitative methodology for evaluating software/system architectures".

These differences are an indicator of the effect of length of experience in software/system development on the responses received from respondents. This is the reason why we chose to use only respondents with above 5 years of experience in the questionnaire, in order to ensure that the opinions and experiences expressed are based on familiarity in the field of system/software development.

Another significant relationship noted was between the respondents who agree that use of models "makes the designers/programmers job much easier" and those who think that "Reliable tools are important for developing/or evaluating software/system architectures". This positive relationship is a confirmation that the identification of the factors encouraging the use of models in software/system description is interdependent, depending on the general preferences of the respondents.

In a similar way, independent sample t-tests Chi-Square and ANOVA tests performed on various hypotheses revealed interesting relationships between various responses, reflecting the wide variety of preferences by the respondents. For example, an independent sample t-test the hypothesis $(\mu>3)$ for the item "Reliable tools are important for developing/or evaluating software/system architectures " showed significance at $5 \%$ level, $\mathrm{t}=2.2, \mathrm{p}$-value $<.05$. This reveals that the population mean of the item "Reliable tools are important for developing/or evaluating software/system architectures" is significantly higher for the respondents who replied "Yes" to the item "It makes the designers/programmers job much easier", compared to those who disagreed.

In a similar way, an independent sample t-test revealed a 
direct relationship between the statements "Current technology allows us to develop general software evaluation models that assess any software architecture against any quality attributes" and "Yes" responses to the item "Reliable modelling tools for describing the architecture exist, which makes the usability factor much easier". The test is significant at $5 \%$ level, $\mathrm{t}=2.0$, $\mathrm{p}$-value $=.049<.05$.

A positive result was similarly noticed between the population mean for the item "Current technology allows us to develop general software evaluation models that assess any software architecture against any quality attributes" and "Yes" response to the item "The wide range of modelling language formality (from informal models to formal), makes the selection of architecture description technique more feasible". The test is significant at $5 \%$ level, $\mathrm{t}=2.1$, $\mathrm{p}$-value $<.05$. Similarly, this was the case between the population mean for the item "Current technology allows us to develop general software evaluation models that assess any software architecture against any quality attributes" and "Yes" responses to the item "Architectural models can be compiled to produce a real functioning software/system with existing modelling languages and tools". The test is significant at $5 \%$ level, $\mathrm{t}=2.5, \mathrm{p}$-value $<.05$.

Once again, the above results point out clearly the relationship in opinion between matters relating to the use of current technology in software/system architecture and evaluation methods, which can aid in clear judgement when implementing the recommendations of the study in the software/system development industry.

Another significant relationship was noted via an independent sample t-test conducted to test the hypothesis $\mu>3$ for the item "There is still vagueness in the current literature concerning the differences between the architecture abstraction and high level design, which causes some confusion and perhaps wastes time during development by architects and designers" and those who admitted that it is "Hard to evaluate architecture models against any stakeholder's quality attributes (e.g. Security, performance)", $\mathrm{t}=-1.96$, $\mathrm{p}$-value $=.028<.05$. This was the case as well for the item "Most of the existing software architecture evaluation methods, produce qualitative results" at $5 \%$ when using a two-tailed test, $(\mathrm{t}=1.94$, $\mathrm{p}$ value $>.05)$.

In order to test the dependence between two categorical variables, a Chi-square test was used. For the relationship between the variables "Usage of software style/pattern concepts \& models during architecture development, increases the utilisation of modelling description languages, BUT decreases the simplicity of the architecture valuation" and "Most of the existing software architecture evaluation methods, produce qualitative results", the Chi-square test showed significance, $\chi^{2}(16)=30.52$, p-value $<.05$. This is proof of an association between the two categorical variables. A similar dependence was deduced between the categorical variables "Usage of software style/pattern concepts \& models during architecture development, increases the utilisation of modelling description languages, BUT decreases the simplicity of the architecture valuation" and "Current technology allows us to develop general software evaluation models that assess any software architecture against any quality attributes" $\chi^{2}(16)=60.10$, pvalue $<.01$.

The above are some of the relationships established between categorical variables, aiding further to deduce the trend in opinion amongst software/system developers in industry with the aim of improving their experience with system/software architecture in the future. In a similar way, other positive relationships were discovered between various pairs of categorical variables including the "awareness of respondents about any system/software architectural tactics or metrics that have been or are being used for evaluating architectural description models" and the opinion of respondents on the statement that "Most of the existing software architecture evaluation methods, produce qualitative results", $\chi^{2}(4)=9.55$, $\mathrm{p}$-value $=.4<.05$, among others highlighted in the full analysis document.

\section{CONCLUSION AND RECOMMENDATION}

Description and evaluation of software/system architecture largely depends on how it has been embraced by developers. This study reveals the great effort that is needed to increase the awareness, knowledge and use of system software architecture in industry, academic institutions, and government sectors.

Various factors that encourage and hinder the embracement of architectural modelling in the software industry were also identified. The researchers recommend further research to determine how to standardize the languages used in architectural modelling to make the models compatible with other models that are already existing. There is also a need to increase awareness of system/software architecture description by documenting more literature on the subject and incorporating it in the curricula of various learning institutions.

\section{REFERENCES}

[1] L. Bass, P. Clements, and R. Kazman, Software Architecture in Practice, 1st ed., ser. SEI Series in Software Engineering. USA: Addison Wesley Longman Inc., 1998.

[2] G. Moreno and P. Merson, "Model-driven performance analysis," in Quality of Software Architectures: Models and Architectures. Springer, 2008, pp. 135-151.

[3] A. Diaz-Pace, H. Kim, L. Bass, P. Bianco, and F. Bachmann, "Integrating quality-attribute reasoning frameworks in the arche design assistant," in Quality of Software Architectures. Models and Architectures. Springer, 2008, pp. 171-188.

[4] G. Zayaraz, "Quantitative approaches for evaluating software architectures," Ph.D. dissertation, Pondicherry Engineering College, Puducherry, India, 2010.

[5] L. Bass, P. Clements, and R. Kazman, Software Architecture in Practice, 3rd ed., ser. SEI Series in Software Engineering. USA: Pearson Education, Inc., 2013.

[6] A. Jaquith, Security metrics: replacing fear, uncertainty, and doubt. Addison-Wesley Upper Saddle River, 2007.

[7] C. Jones, Applied software measurement: global analysis of productivity and quality. Mcgraw-hill New York, 2008, vol. 3. 